\title{
Vortex Pattern on a Fixed Reynold's Number with Varying Aspect Ratio, and Vice Versa in a Lid Driven Square Cavity
}

\section{ALIYU A. Baba', BELLO Zubairu² and SUNDAY Ayigun ${ }^{3}$}

${ }^{1}$ Department of Mechanical Engineering, Federal polytechnic Mubi. Adamawa State.

${ }^{2}$ Department of Chemical Engineering, Federal polytechnic Mubi. Adamawa State.

${ }^{3}$ Department of Science Laboratory Technology, Federal polytechnic Mubi. Adamawa StateNigeria.

\section{ARTICLE INFO}

Article No.: 061718084

Type: Research

DOI: 10.15580/GJSETR.2018.2.061718084

Submitted: $17 / 06 / 2018$

Accepted: $22 / 06 / 2018$

Published: 27/06/2018

${ }^{*}$ Corresponding Author

Aliyu A. Baba

E-mail: adamuldam@ yahoo.com

\section{Keywords:}

lid driven square cavity, Reynold's

number, Aspect ratio, Vortex
The modelling of flow for laminar fluid in lid driven square cavity was conducted by employing a code that run in MATLAB and in the first simulation, a fixed value of the Reynold's number of 600 was maintained, it was observed from the figures and results that as the aspect ratios were varied (increased) from $0.3,0.4$ and 0.6 , the vortex tends to increase in number of streamlines closing the spaces between each other and widening to occupy the bottom right. However, in the second simulation, where the aspect ratio of 0.2 was maintained and varying different Reynolds numbers of 400,500 and 600 , the vortex formed also increases and becoming too close to one another as the Reynold's number increases, thereby indicating that the pressure coefficient inside the lid square cavity is controlled by the aspect ratio as well as the Reynold's number. 


\section{INTRODUCTION}

Fluid dynamics has been generally accepted as an aid to solving Navier Stokes equation and a significant tool for comprehending the physics of numerous complex fluid flow analyses (Gokhale and Fernandes, 2014). However, lid driven cavity flow is the fluid motion within a rectangular/square cavity formed from one side being at rest and a constant translational velocity of the other side. Lid driven cavities have numerous applications, which include dynamics of lakes, material processing metal galvanizing and casing (Omari, 2013). It was stated in (Omari, 2013) that flow through cavity shows characteristics that is likely to happen in incompressible flows: secondary flows, eddies, chaotic particle motions, instability, complex 3-dimension and turbulence.

Furthermore, a computational fluid dynamics (CFD) simulation conducted by Omari for laminar incompressible fluid flow in lid driven cavity $(10 \leq R e \leq$ 1000 ) with height to width ratio ranging from 0.5 to 1.5 and given in terms of stream contours, pressure coefficient and velocity profiles indicated that the pressure coefficient inside the cavity is predominantly controlled by Reynolds number as well as the aspect ratio. Flow inside a cavity is also used as a model problem to study bifurcation, flow stability and transition to turbulence through direct numerical simulations (Peng, Shiau \& Hwang, 2003; Bruneau \& Saad, 2006).

Kosti, 2015 investigated varying Reynold's values and grid sizes and observed that Peaks of the profiles of the velocity increases with an increase in the value of the Reynold's number.

Consequently, this study is on the determination of the effect on the vortex/stream pattern of varying Reynold's numbers with a fixed aspect ratio, and varying aspect ratios with a fixed Reynold's number in a lid driven square cavity.

\section{Theory Formulation}

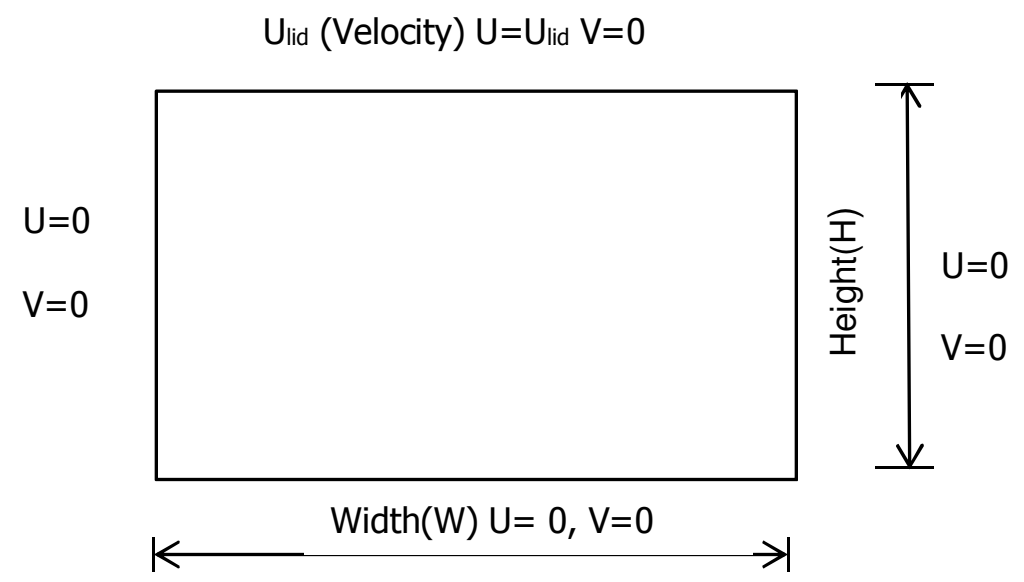

Figure 1: Schematic of a Square Cavity

In the flow in a square cavity driven by shear force, the flow will be governed by two dimensionless parameter $\mathrm{W} / \mathrm{H}$.

$$
\begin{aligned}
\text { Reynold Number } & =\frac{U_{\text {lid }} \times H}{\frac{\mu}{\rho}} \\
& =\frac{U_{l i d} \times H}{v}
\end{aligned}
$$

Where, $\mathrm{W}$ and $\mathrm{H}$ are the cavity width and cavity height. Ulid is the reference velocity dimension, $v$ is the kinematic viscosity, $\mu$ is the coefficient of dynamic viscosity and $\rho$ is the density of the fluid.

The vorticity equation is given as:

$$
\begin{gathered}
w=\frac{\partial v}{\partial x}-\frac{\partial u}{\partial y} \\
=-\left(\frac{\partial^{2} \tau}{\partial x^{2}}+\frac{\partial^{2} \tau}{\partial y^{2}}\right)
\end{gathered}
$$

Where, $u$ and $v$ are the velocities in the $\mathrm{x}$ and $\mathrm{y}$ directions, $w$ is the vorticity, $\tau$ is the stream function.

$$
\begin{aligned}
w_{i, j}= & -\left\{\frac{\left(\tau_{i+1, j}-2 \tau_{i, j}+\tau_{i-1, j}\right)}{\Delta \mathrm{x}^{2}}\right. \\
& \left.+\frac{\left(\tau_{i, j+1}-2 \tau_{i, j}+\tau_{i, j-1}\right)}{\Delta \mathrm{y}^{2}}\right\} \quad 3
\end{aligned}
$$

where $\mathrm{i}$ and $\mathrm{j}$ are values of $\mathrm{x}$ and $\mathrm{y}$ nodes starting from $\mathrm{i}$ $=1,2, \ldots$ and $j=1,2, \ldots$.

Equation 3 above is the vorticity equation in discretised form and from it, we have: 


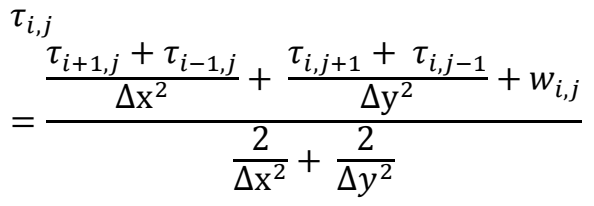

\section{METHODOLOGY}

The formula for Reynolds Number in equation 1 was used for two cases:

1. A fixed value of the Reynold's number was maintained while three different values of the aspect ratio was varied with a consequential change of $\bigcup_{\text {lid }}\left(\bigcup_{\text {top }}\right)$.

2. A fixed value of the aspect ratio was used while varying the value of the Reynold's number, which also changes the $\bigcup_{\text {lid }}\left(\bigcup_{\text {top }}\right)$.

A fixed value of the Reynold number 600 was kept constant and three different values of the aspect ratios
$(\mathrm{W} / \mathrm{H})$ varied in the MATLAB code, by varying the aspect ratio, the $U_{\text {top }}$ in the MATLAB code was also varied and the code run for $0.3,0.4$ and 0.6 values.

Fixed value of aspect ratio 0.2 was used, the same value of 0.2 was maintained for both the height and width because we are considering a flow in a square lid driven cavity while the Reynold's numbers of 400, 500 and 600. As this was performed and varied in the MATLAB code, the program was run and the plots saved and analysed.

\section{BOUNDARY CONDITIONS}

There is no slip velocity at the wall; right, left and bottom velocity are considered as zero (i.e $u=0 \& v=0$ for the right, left and bottom walls). On the lid of the cavity, there will be value of the top velocity.

In order to implement the equation into MATLAB, the vorticity transportation equation obtained need to be converted into a discretized equation.

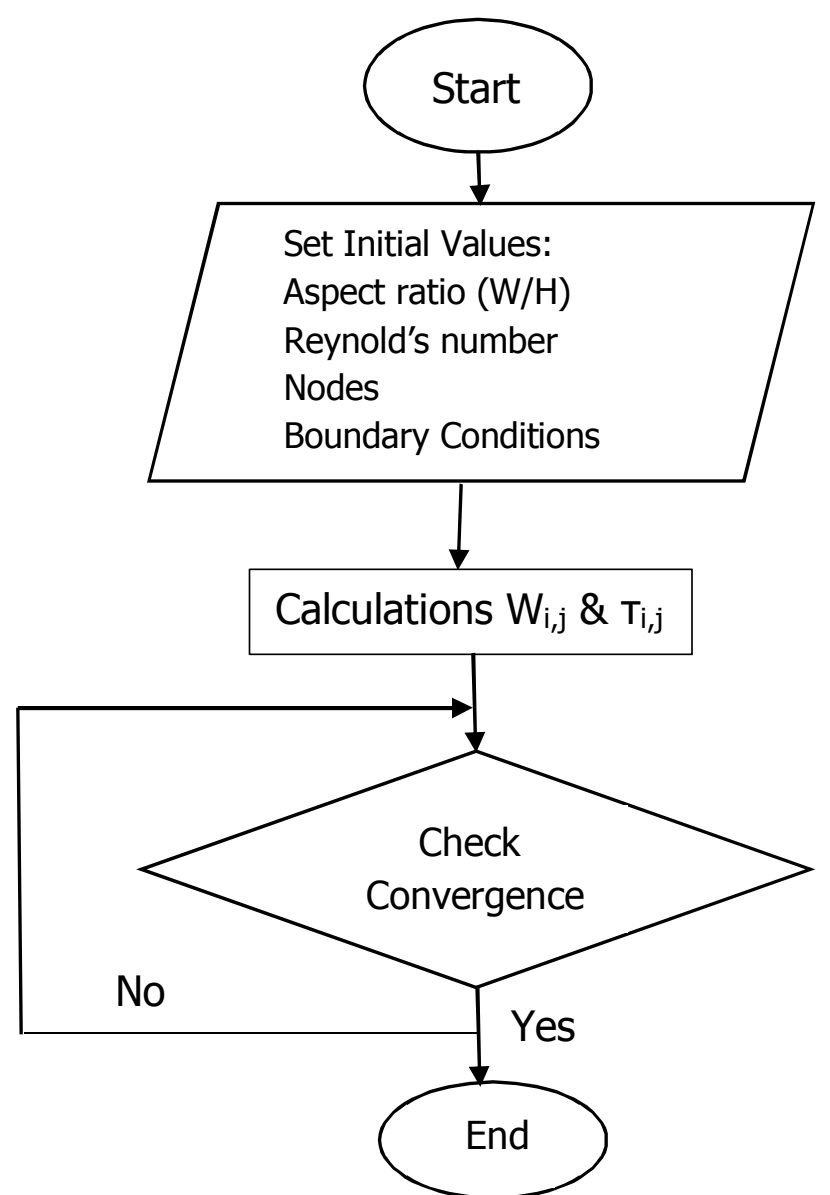

Figure 2: Flowchart of the brief algorithm in MATLAB

Tables I and 2 below show the various values of the Reynold's numbers and aspect ratios used to run the simulation. A convergence criterion of $1 \times 10^{-6}$ was applied in this study. 
Table 1: Fixed Reynolds Number of 600 with varying aspect ratio

\begin{tabular}{lcc}
\hline S/No. & Aspect ratio & $U_{\text {top }}$ \\
\hline 1 & 0.30 & 0.05 \\
2 & 0.40 & 0.038 \\
3 & 0.60 & 0.025 \\
\hline
\end{tabular}

Table 2: Fixed Aspect ratio of 0.2 with Varying Reynold's Number

\begin{tabular}{lcc}
\hline S/No. & Reynolds Number & $U_{\text {top }}$ \\
\hline 1 & 400 & 0.05 \\
2 & 500 & 0.063 \\
3 & 600 & 0.075 \\
\hline
\end{tabular}

\section{RESULTS AND DISCUSSION}

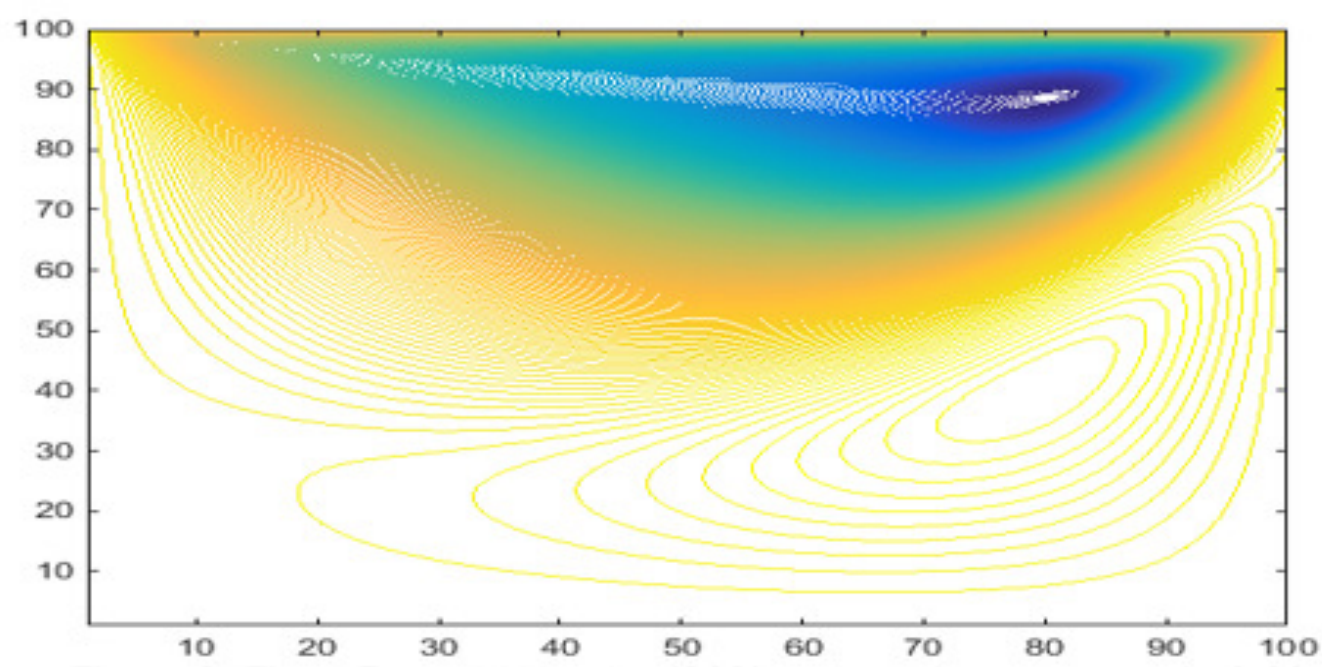

Figure 3: Fixed Reynold's number of 600 and Aspect ratio of 0.3

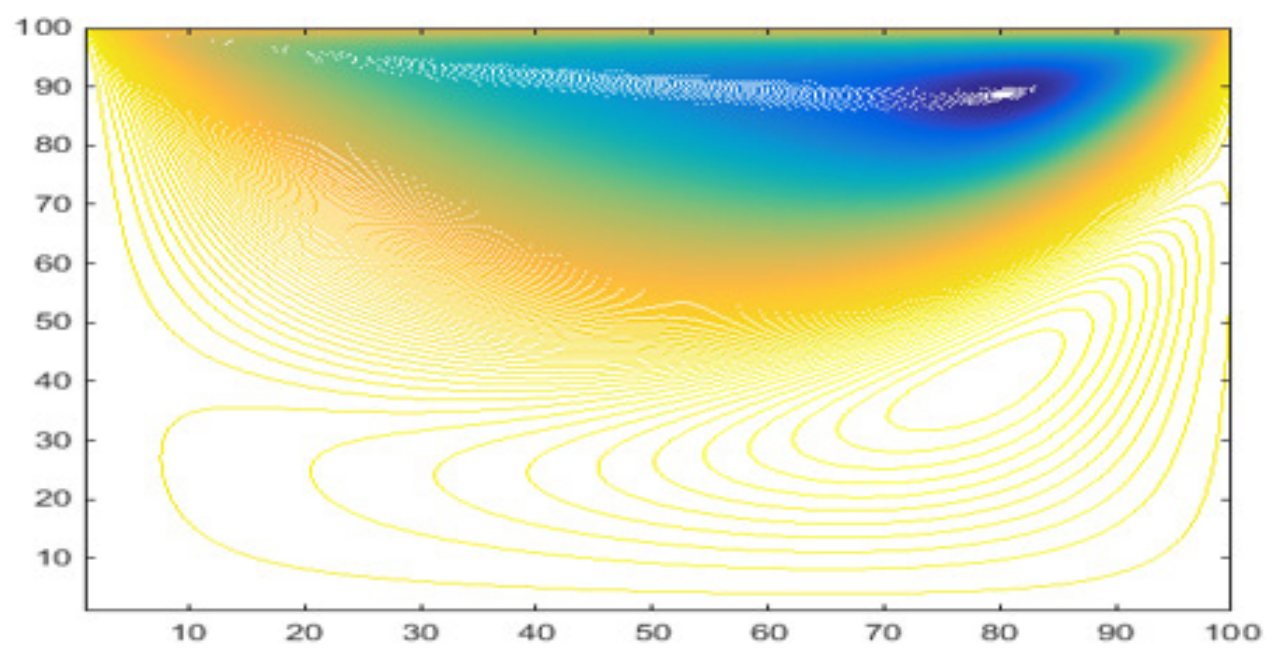

Figure 4: Fixed Reynold's number of 600 and Aspect ratio of 0.4 


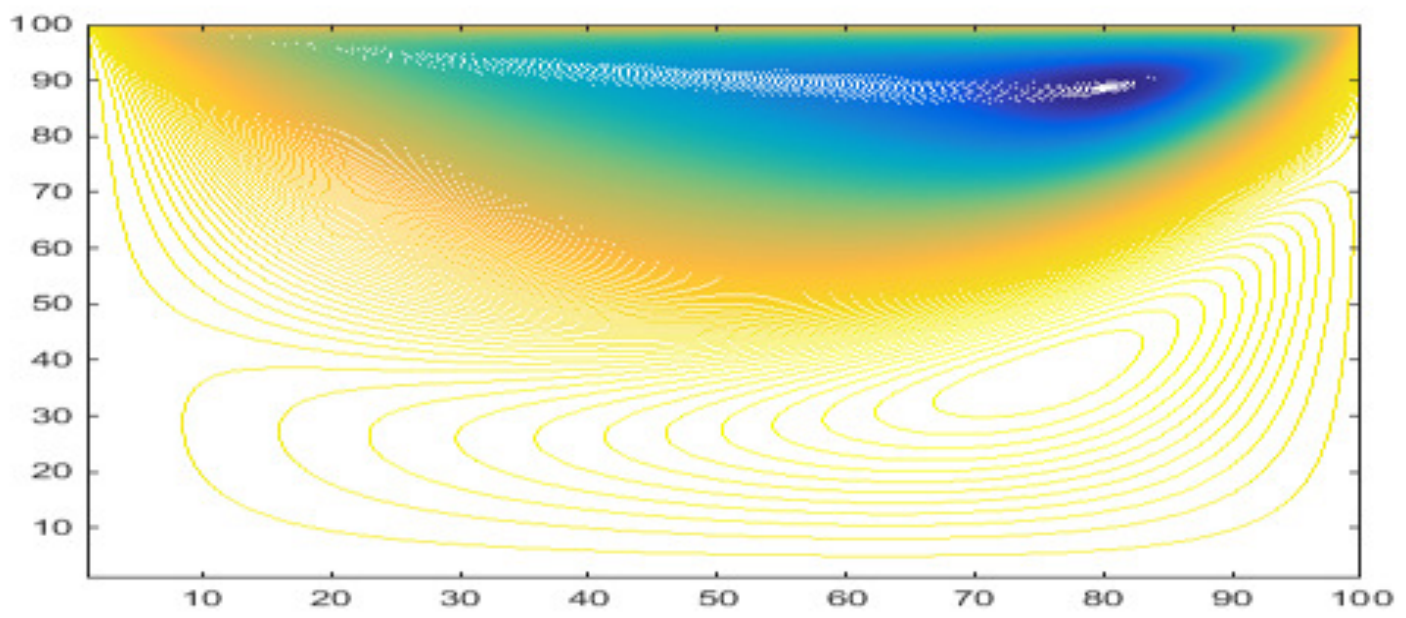

Figure 5: Fixed Reynold's number of 600 and Aspect ratio of 0.6

Figures 3, 4 and 5 represent the contours of stream lines at the top and the vortex formed at the bottom, for different aspect ratios while the Reynolds number of 600 is kept constant. It was observed from the figures that as the aspect ratios were varied (increased) from 0.3, 0.4 and 0.6 ; the vortex tends to increase in number of streamlines closing the spaces between each other and widening to occupy the bottom right. At $\operatorname{Re}=400,500$ \& 600 the development of a second and subsequent vortices shape streamlined at the right bottom of the cavity was observed and was expanding with increase in Reynold's number.

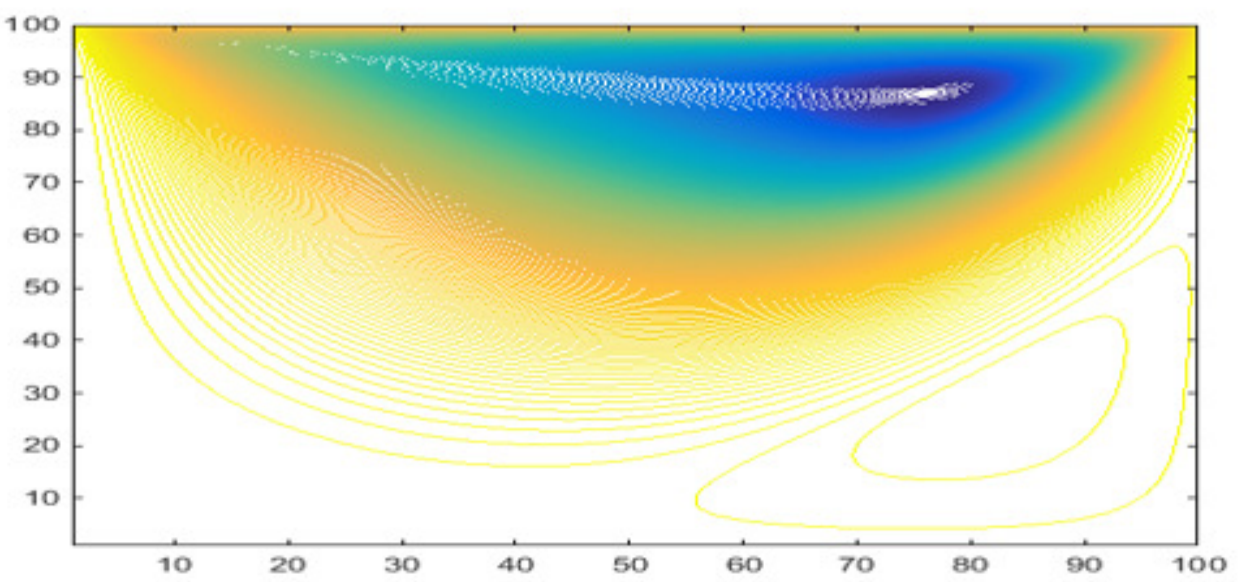

Figure 6: Fixed Aspect ratio of 0.2 and varying Reynold's number of 400 from Utop

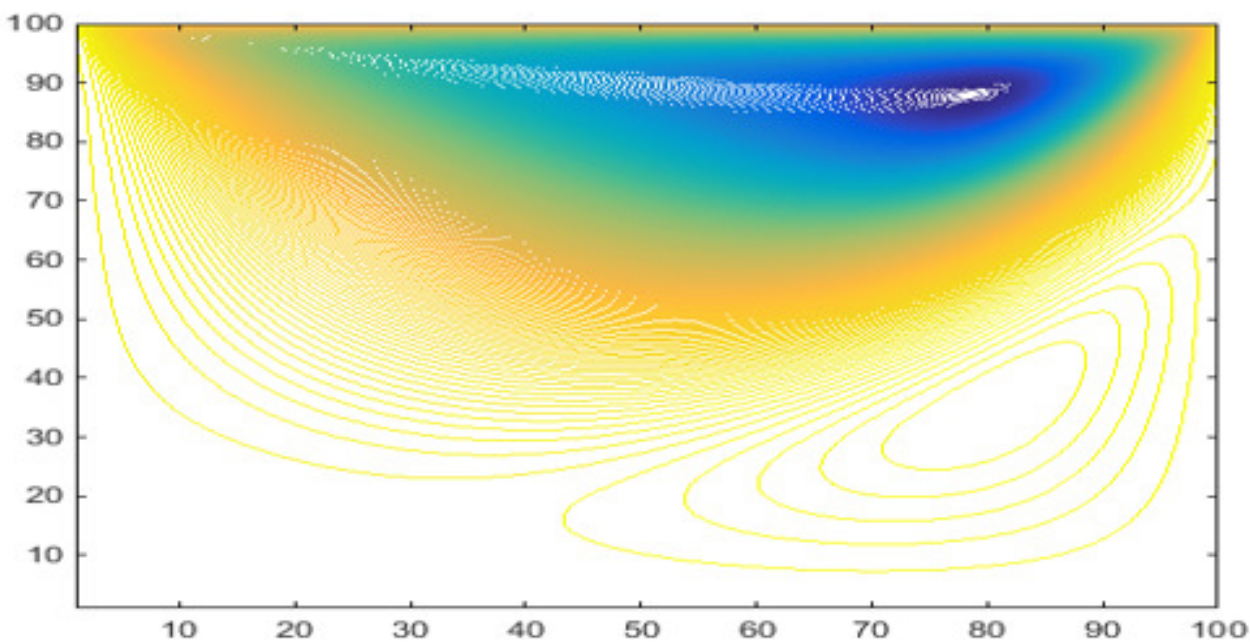

Figure 7: Fixed Aspect ratio of 0.2 and varying Reynold's number of 500 from Utop 


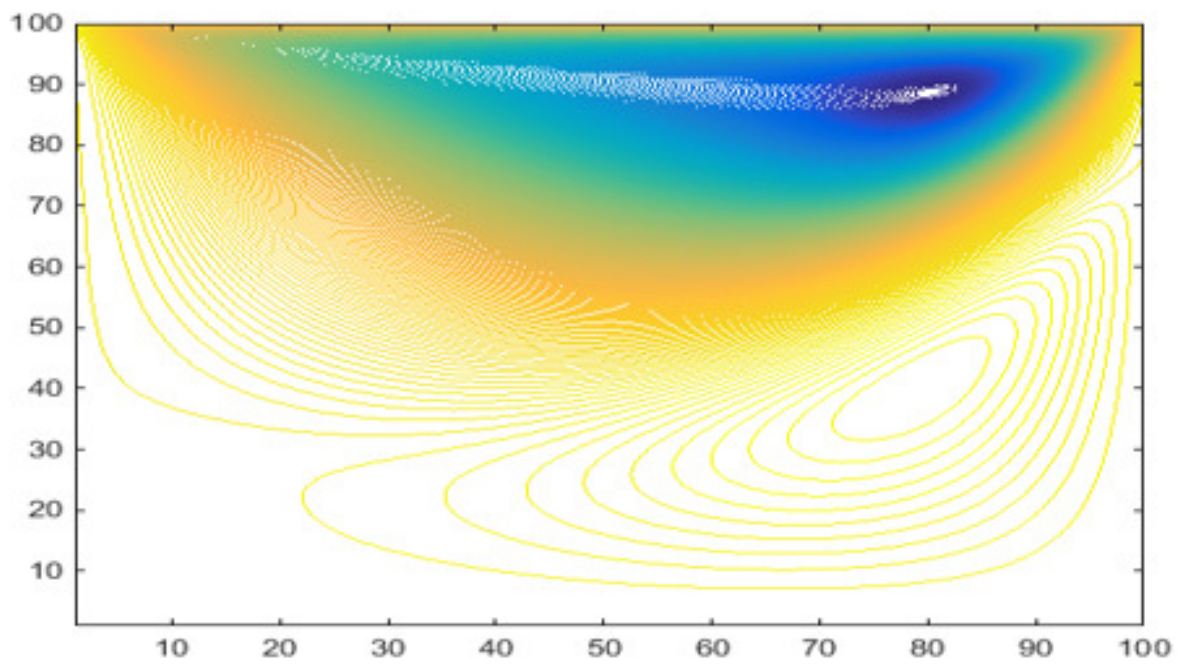

Figure 8: Fixed Aspect ratio of 0.2 and varying Reynold's number of 600 from Utop

Figures 6, 7 and 8 show the vortex formed with different Reynolds numbers of 400,500 and 600 and a fixed aspect ratio of 0.2 . The figures of the results indicated that, as the Reynolds number is increased and maintaining a fixed aspect ratio, the vortex also increased in numbers and becoming too close to one another. The size of right corner eddies kept increasing as the value of aspect ratio is increased. In summary, the operation of different aspect ratios affects the development of streamline, the size of primary and corner eddies. As the value of aspect ratio is increased, the size of primary eddies decreases, the size of left corner decreases while the size of right corner eddies increases.

\section{CONCLUSION}

From the pattern and formation of the vortex in the simulation, it is evident that the Reynolds number and the aspect ratios are very important parameters in determining the vortex that could be formed in a lid driven square cavity. With an increase in either the Reynolds number or aspect ratio, there is always an increase in the size of the vortex created or produced.
The obtained results showed similar output with that of other literatures studied.

\section{REFERENCES}

Gokhale, M. Y. and Fernandes, I. (2014). Lattice boltzmann simulation of fluid flow in a lid driven cavity. International Journal on Mechanical Engineering and Robotics, 2 (1), 1-5.

Omari, R. (2013). CFD simulations of lid driven cavity flow at moderate Reynolds number. European Scientific Journal, 9, (15), 22-35.

Peng, Y.F., Shiau, Y.H., and Hwang, R.R. (2003). Transition in a 2-D lid-driven cavity flow, Comput. Fluids, 32, 337-352.

Bruneau, Ch. H. and Saad, M. (2006). The 2D lid-driven cavity problem revisited, Comput. Fluids, 35, 326348.

Kosti, S. and Rathore, V. S. (2015). Numerical Study of Lid Driven Cavity at Different Reynolds Number. Trends in Mechanical Engineering \& Technology. 5. 42-46. 


\section{Appendix}

MATLAB code considered in this study

Fixed Reynold Number $=600$
Height $=0.6$
Width $=0.6$
Utop $=0.025$

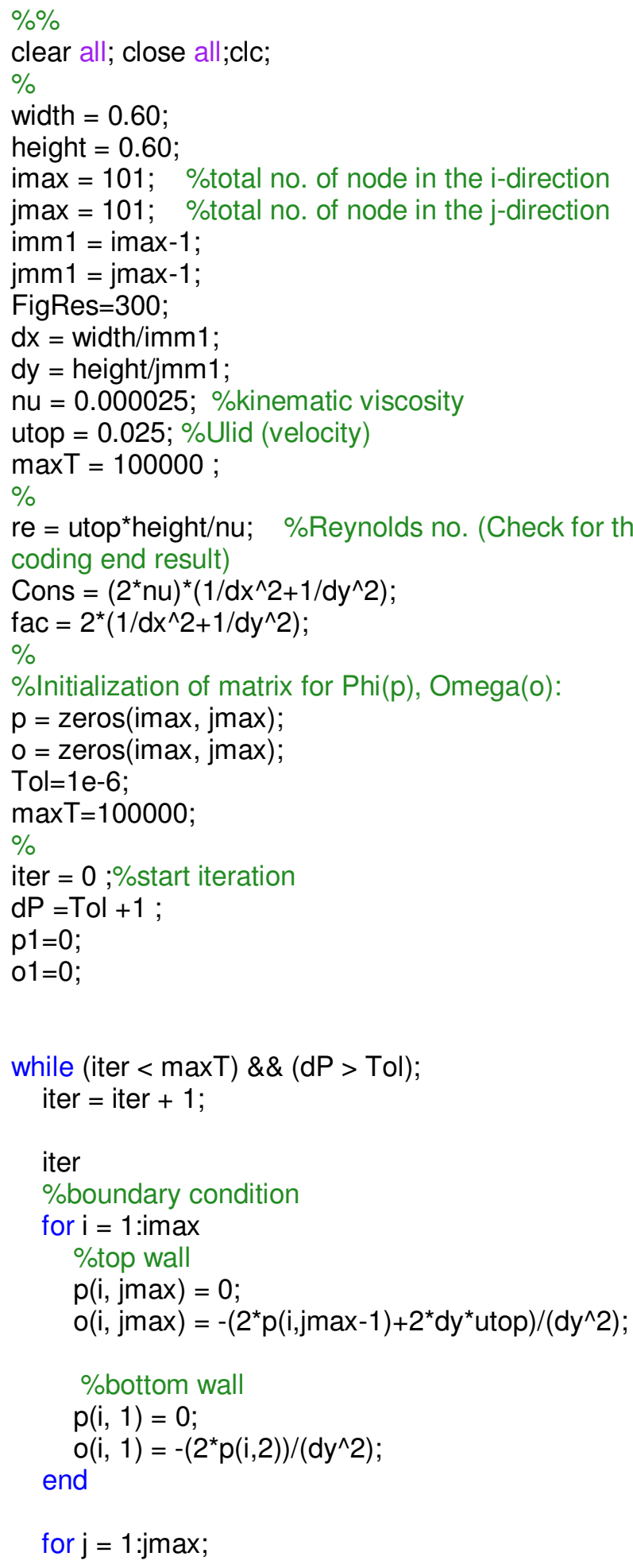




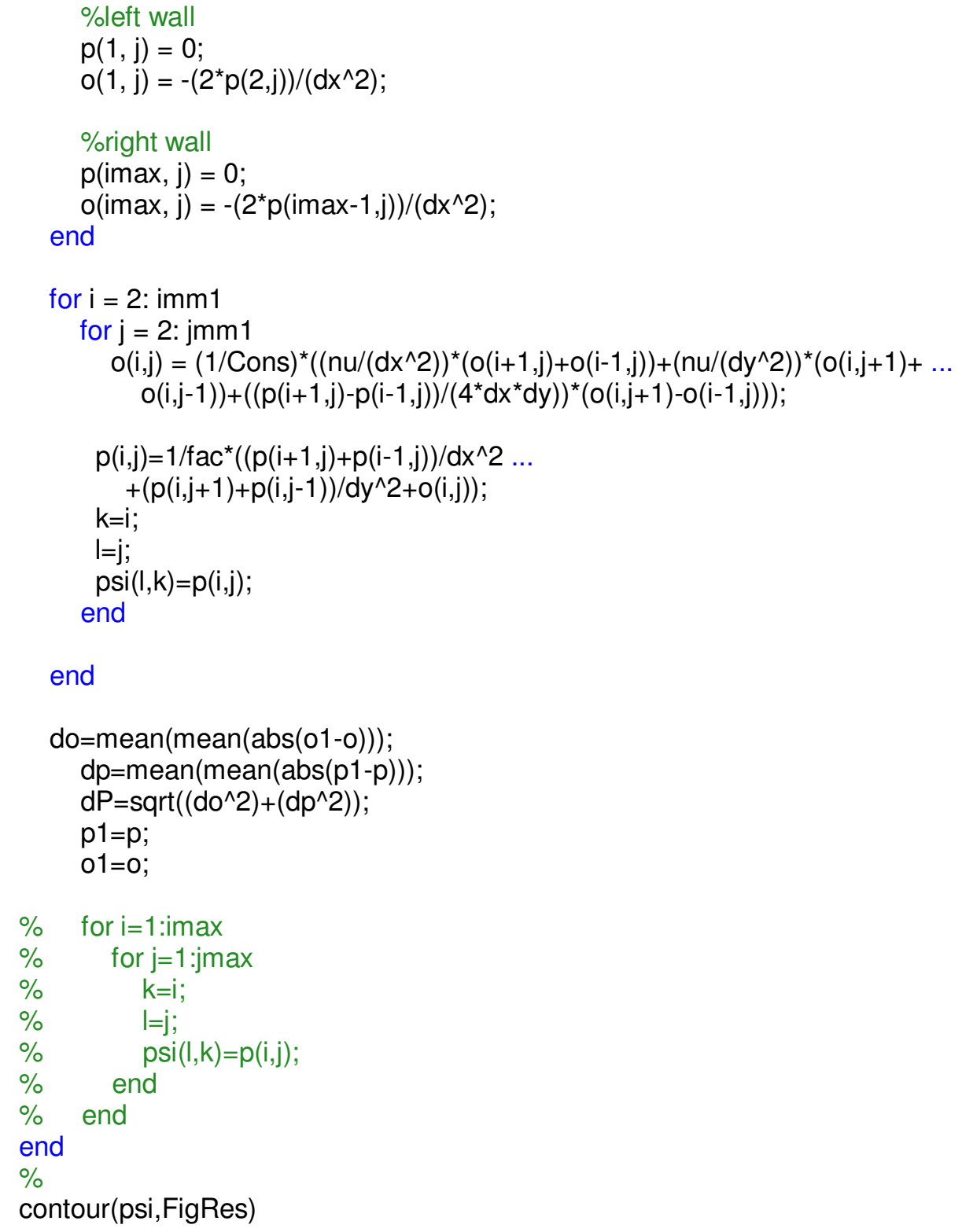

